\title{
Cux1 and Cux2 Selectively Target Basal and Apical Dendritic Compartments of Layer II-III Cortical Neurons
}

\author{
Beatriz Cubelos, ${ }^{1}$ Carlos G. Briz, ${ }^{2}$ Gemma María Esteban-Ortega, ${ }^{2}$ Marta Nieto $^{2}$ \\ ${ }^{1}$ Departamento de Biología Molecular, Centro de Biología Molecular 'Severo Ochoa', \\ Universidad Autónoma de Madrid, UAM-CSIC, Nicolás Cabrera, 1, Madrid 28049, Spain \\ ${ }^{2}$ Department of Molecular and Cellular Biology, Centro Nacional de Biotecnología, CNB-CSIC, Darwin 3 , \\ Campus de Cantoblanco, Madrid, 28049 Spain
}

Received 23 April 2014; revised 9 July 2014; accepted 21 July 2014

\begin{abstract}
A number of recent reports implicate the differential regulation of apical and basal dendrites in autism disorders and in the higher functions of the human brain. They show that apical and basal dendrites are functionally specialized and that mechanisms regulating their development have important consequences for neuron function. The molecular identity of layer II-III neurons of the cerebral cortex is determined by the overlapping expression of Cux1 and Cux2. We previously showed that both Cuxl and $C u x 2$ are necessary and nonredundant for normal dendrite development of layer II-III neurons. Loss of function of either gene reduced dendrite arbors, while overexpression increased dendritic complexity and suggested additive functions. We herein characterize the function of Cuxl and Cux2 in the development of apical and basal dendrites. By
\end{abstract}

in vivo loss and gain of function analysis, we show that while the expression level of either Cux1 or Cux2 influences both apical and basal dendrites, they have distinct effects. Changes in Cuxl result in a marked effect on the development of the basal compartment whereas modulation of Cux2 has a stronger influence on the apical compartment. These distinct effects of $\mathrm{Cux}$ genes might account for the functional diversification of layer II-III neurons into different subpopulations, possibly with distinct connectivity patterns and modes of neuron response. Our data suggest that by their differential effects on basal and apical dendrites, Cuxl and Cux2 can promote the integration of layer II-III neurons in the intracortical networks in highly specific ways. () 2014 Wiley Periodicals, Inc. Develop Neurobiol 75: 163-172, 2015 Keywords: cortex; cut; CDP; dendrite; autism

\section{INTRODUCTION}

Development of dendritic structures is an important facet of circuit regulation in the cerebral cortex. It is critical for the establishment of specific networks

Additional Supporting Information may be found in the online version of this article.

Correspondence to: B. Cubelos (bcubelos@cbm.csic.es) and M. Nieto (mnlopez@cnb.csic.es).

Contract grant sponsor: Ministerio de Ciencia e Innovación (MICINN); contract grant numbers: SAF2011-23735 (to M.N.), SAF2012-31279 (to B.C.), and RyC-2010-06251 (to B.C.).

(C) 2014 Wiley Periodicals, Inc.

Published online 24 July 2014 in Wiley Online Library (wileyonlinelibrary.com).

DOI 10.1002/dneu.22215 during differentiation and for decoding and transmitting electrical signals in mature circuit (Cline and Haas, 2008; Jan and Jan, 2010; Kulkarni and Firestein, 2012). Dendrites of pyramidal neurons are morphologically and functionally divided into basal and apical compartments (Barnes et al., 2008). Subcellular polarization is established early in migrating neurons, is maintained throughout the life of the cell, and is reflected in the asymmetric distribution of several cytoplasmic molecules and membrane receptors such as the alpha5 subunit of the integrin adhesion receptor VLA5 (Bi et al., 2001) and the neuropilin (Nrp) receptor 2 in the apical processes (Tran et al., 2009).

The apical and basal regions of the dendritic arbor are functionally specialized, and evidence shows that 
modifications in dendrite branch number or length in any of these compartments have important and selective consequences for neuron and circuit function (Niblock et al., 2000; Polleux et al., 2000; Tran et al., 2009; de Anda et al., 2012; Srivastava et al., 2012b; Miao et al., 2013). Basal and apical dendritic topology and length contribution to neurons' functions include facilitation of contact with potential axonal inputs, which are organized in layers in the cerebral cortex, as well as the restriction of the number of potential synapses (Tada and Sheng, 2006; Parrish et al., 2007; Shen and Scheiffele, 2010). Propagation of electrical signals also differs in distal apical and basal domains, and thus regulation of these compartments has selective consequences in input computation and in the amplification or silencing of presynaptic depolarization signals (Mainen and Sejnowski, 1996; Jia et al., 2010; Branco and Hausser, 2011; Feldman, 2012). A striking number of recent reports implicate mechanisms that regulate apical and basal development in autism disorders, highlighting not only the existence of these pathways but also their importance for higher functions of the human brain (de Anda et al., 2012; Srivastava et al., 2012b; Miao et al., 2013).

Layer II-III neurons characterize the mammalian cortex, show complex dendritic arbors, and participate in highly interconnected intracortical circuits involved in associative functions. At molecular level, their identity is determined by the selective expression of Cuxl and Cux2 (Nieto et al., 2004). These homeodomain proteins positively regulate dendritic growth and facilitate layer II-III neuron ability to integrate information from multiple axonal inputs, enabling their specialized tasks (Cubelos et al., 2010). Cuxl and Cux2 genes have very similar expression patterns, and both proteins are coexpressed in most superficial neurons, although not at equal levels (Nieto et al., 2004; Zimmer et al., 2004). Our earlier studies showed that, despite their similar expression and structure, both Cux proteins are necessary to sustain normal dendritic development of layer II-III neurons in a nonredundant manner; we demonstrated that their combinatorial expression is responsible for the distinctive complex dendritic arbor of these neurons (Cubelos and Nieto, 2010; Cubelos et al., 2010). The mechanisms responsible for these complementary functions remain unknown.

The relevance of apical and basal dendrites in neuron function suggests that the selective control of these compartments might account for the nonredundant functions of Cuxl and Cux2. We, therefore, analyzed the specific changes in the apical and basal dendritic arbor that resulted from altering $\mathrm{Cux}$ expression. We found that Cuxl and Cux2 have distinct effects on the topology of superficial neurons. Modifying Cuxl or Cux2 expression affected both apical and basal branching, however, the effects of Cuxl were stronger on basal dendrites, whereas modifying Cux2 levels mainly altered apical dendrites. These results confirm the nonredundant functions of the two mammalian $C u x$ paralogs and clarify their complementary functions on the dendritic topology of layer II-III neurons. It also defines a mechanism that might functionally diversify layer II-III neurons into different subpopulations, possibly with different connectivity patterns and modes of neuronal response.

\section{METHODS}

\section{Animals}

All animal procedures were approved by the Centro Nacional de Biotecnología Animal Care and Use Committee $\left(\mathrm{N}^{\mathrm{O}}\right.$ 11001), in compliance with national and European Union legislation. $\mathrm{Cu} \times 2^{-/-}$mice (C57BL6 background) have been described (Cubelos et al., 2008a). For overexpression, C57BL6 wild type (WT) mice (Harlan Laboratories) were used. The morning of the day of appearance of the vaginal plug was defined as embryonic day (E) 0.5 .

\section{In Utero Electroporation and GFP Immunohistochemistry}

In utero electroporation was as described (Tabata and Nakajima, 2001). shRNACuxl, CAG-Cuxl, and CAG-Cux2 (Cubelos et al., 2010) or empty pCAG used as control plasmids $(1 \mu \mathrm{g} / \mu \mathrm{L})$ were mixed with $p C A G-G F P(1 \mu \mathrm{g} / \mu \mathrm{L})$. Embryos were electroporated at E15.5. Electroporated mice were perfused at $\mathrm{P} 21$ with phosphate-buffered saline followed by a neutral-buffered $10 \%$ formalin solution (Sigma) (Cubelos et al., 2008a). Brains were postfixed overnight, embedded in sucrose and cryostat sectioned. Fifty micrometer sections with electroporated neurons were stained with anti-GFP (A-11122, Molecular Probes).

\section{Confocal Microscopy and Morphological Analysis}

Confocal microscopy was performed with a Bio-Rad Radiance 2100 laser scanning Zeiss axiovert 200 microscope (argon $488 \mathrm{~nm}$ laser line for excitation). After sectioning and staining, individual neurons of the somatosensory cortex were analyzed by obtaining $0.2 \mu \mathrm{m}$ serial optical sections using confocal and Lasaf v1.8 software (Leica). Measurements were only made of neurons with the main apical process parallel to the plane of section that contacted layer I. Confocal sections were used to reconstruct neuron morphology using Lasaf v1.8 software. Dendritic processes were measured in the planar projection with LaserPix (Image-Pro Plus v.4.0, BioRad; see Supporting Information Fig. 1) and exported to excel file to process the data. The 
cumulative dendritic length of total branches, and the number and cumulative length of primary, secondary, and tertiary branches was calculated.

\section{Statistical Analysis}

All results are expressed as the mean \pm SEM. Experimental groups were compared with one-way ANOVA followed by Tukey's post hoc test. Test Student's two-sample $t$ test. $p$ values are indicated in figure legends.

\section{RESULTS}

\section{Loss of Cux2 Function Mainly Affects Apical Dendrites}

Our earlier studies demonstrated that overexpression of either Cuxl or Cux2 promote dendritogenesis, and in vivo knock-out and knock-down experiments showed that both genes have necessary functions for normal dendrite development (Cubelos et al., 2010). The nonredundant functions of Cux proteins suggest differential effects possibly on distinct neuronal compartments. To study this possibility, we analyzed the basal and apical dendrites of layer II-III neurons of the somatosensory cortex in WT and $\mathrm{Cux} 2^{-/-}$mice; $C A G$ GFP ( $C M V$ immediate enhancer/ $\beta$-actin promotergreen fluorescent protein) was electroporated in utero at embryonic day (E) 15.5 to label precursors that generate superficial neurons. Morphology of the targeted neurons was analyzed at postnatal day (P) 21, when dendrite development is complete. The total length of all dendrite processes was assessed as a measure of dendritic complexity, and the numbers and length of the primary, secondary, and tertiary branches were quantified. As previously reported, layer II-III neurons of the somatosensory cortex showed an elaborate dendritic morphology that was greatly decreased in $\mathrm{Cux}^{-/-}$neurons [Fig. 1(a,b)]. Our measurements showed reduction in the total cumulative length of apical and basal dendrites in $\mathrm{Cux} 2^{-/-}$compared to control mice; the apical compartment was more profoundly affected, with a $>50 \%$ reduction [Fig. 1(c)]. This was due to a notable decrease in the number of secondary and tertiary apical branches [Fig. 1(d)], whose length did not change [Fig. 1(f)]. In contrast, in the basal compartment, only the number of $\mathrm{Cux}^{-/-}$primary processes was slightly reduced compared to control neurons [Fig. 1(e)]. Morphology of shRNACux2 electroporated layer II-III neurons was undistinguishable from GFP neurons of the Cux $2^{-/-}$. This confirms previous results and validates the interchangeable use of these loss of function strategies (Supporting Information Fig. 2 and Cubelos et al., 2010).
Apical and basal dendrites of cortical neurons contact different axonal inputs, and presynaptic contacts influence dendrite development. Similarly, several secreted factors modulate dendrite development. To elucidate if the observed effects were a non-cell autonomous consequence of a possible undetected defect in $\mathrm{Cux}^{-/-}$mice, we overexpressed $\mathrm{Cu} \times 2$ into layer II-III neurons of the somatosensory cortex of

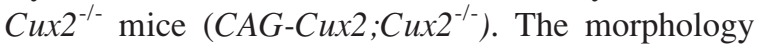
of the targeted neurons was analyzed at P21. Our measurements showed an increase in the total cumulative dendritic length in $C A G-C u \times 2 ; C u \times 2^{-/-}$compared to neurons in $\mathrm{Cux} 2^{-/-}$mice [Fig. 2(a,b)]. This was due to a notable increase in the number of secondary and tertiary apical branches [Fig. 2(c,d)], whose length also slightly increased [Fig. 2(f)]. Com-

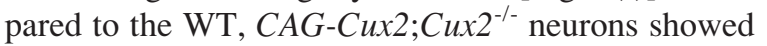
a minor but significant increase in apical dendrites [Fig. 2(c,d)]. Compared to the $C u x 2^{-/-}$, there was an increase in the basal compartment, but it was of lesser magnitude [Fig. 2(c,e)]. CAG-Cux2;Cux2 ${ }^{-/-}$basal dendrites were undistinguishable of the WT control neurons [Fig. 2(c,e,g)]. These data suggests that overexpression using CAG-Cux2 constructs results in higher levels of Cux2 protein that those found in WT neurons. Rescue and increase of the dendritic phenotypes of $\mathrm{Cux} 2^{-/-}$by $\mathrm{Cux} 2$ overexpression, and the stronger effect $C A G-C u x 2$ on the apical compartment, indicates cell autonomous effects independent of other possible defects in the $\mathrm{Cux2^{-/- }}$ cortex. Taken together, data indicates a cell-autonomous preferential targeting of apical dendrites by $\mathrm{Cu} 2 \mathrm{2}$.

\section{Loss of Cux1 Function Affects both Apical and Basal Dendrites}

Analysis of $\mathrm{CuxI}^{-/-}$mice was precluded because they die early after birth due to defects unrelated to the nervous system. Homozygous mutant mice have stunted growth and a high postnatal death rate (Luong et al., 2002; Cubelos et al., 2008b), and we do not recover any animals pasted $\mathrm{P} 0$ on our current colony. To obviate this prenatal lethality, we used in utero electroporation technique, similarly to our previous study (Cubelos et al., 2010). In addition in utero electroporation allows us to study $C u x$ function in a WT context, with most axonal inputs unaffected, and to evaluate cell-autonomous mechanisms (Cubelos et al., 2008b). We electroporated previously characterized shRNA lentiviral constructs that specifically target Cuxl, together with the CAG-GFP plasmid at E15.5 and analyzed layer II-III neurons of the P21 somatosensory cortex. Cuxl down-modulated neurons showed a less elaborate dendritic morphology compared to WT 
a
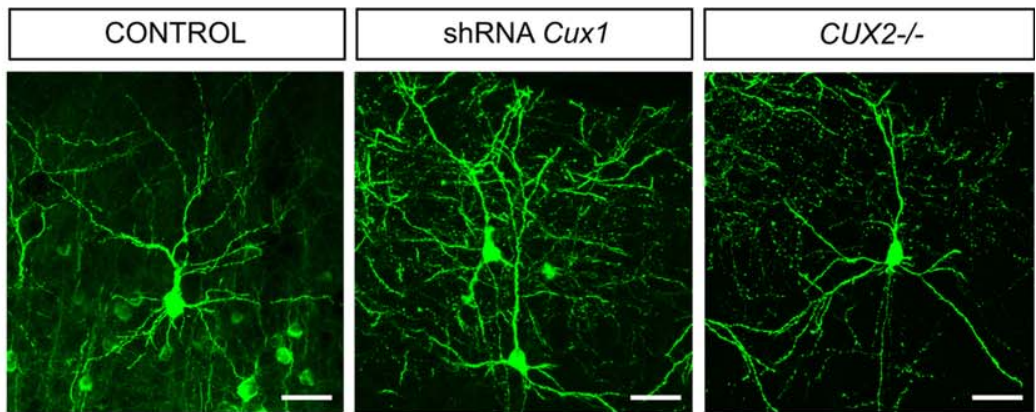

b

Total dendrite length

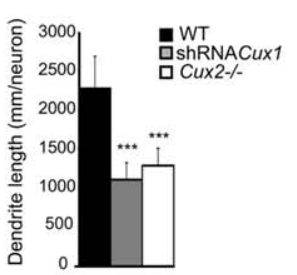

C

Dendrite length

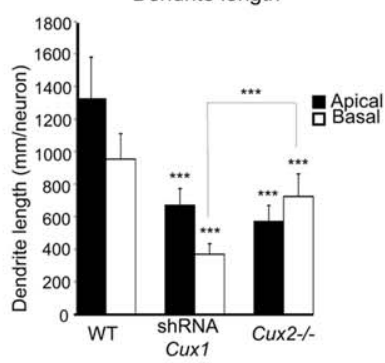

d Number of

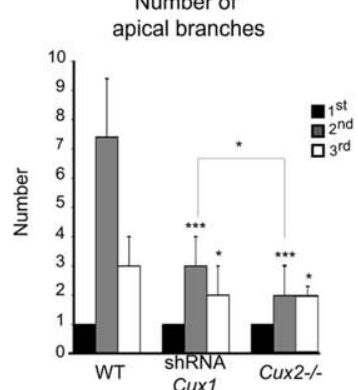

e

Number of

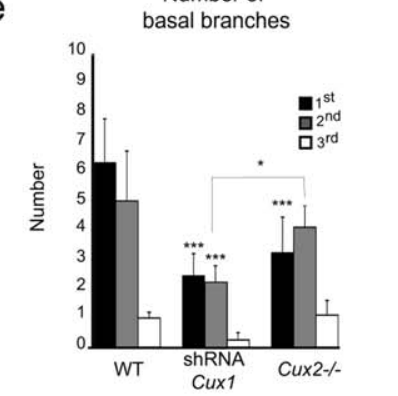

f

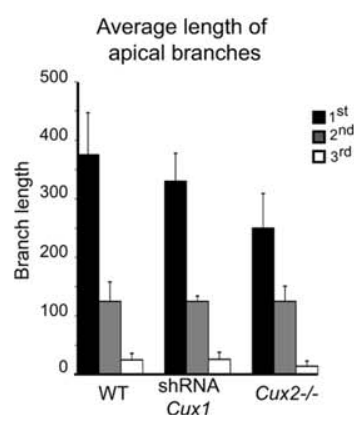

g

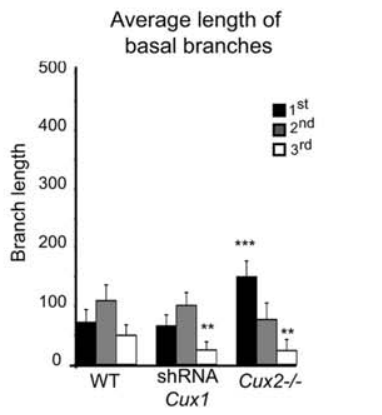

Figure 1 Cux deficient neurons show reduced dendritic tree in the somatosensory cortex. (a) Representative confocal micrographs showing GFP-expressing neurons in the P21 somatosensory cortex in control, shRNACuxl, and $C u x 2^{-/-}$mice. Neuron morphology was analyzed after in utero electroporation of E15.5 cortical precursors. The morphology of pyramidal neurons in upper cortical layers is simpler, shorter, and with fewer dendritic branches in neurons targeted with shRNACuxl and in upper layer neurons of $C u \times 2^{-/-}$than in WT mice. Bar $=50 \mu \mathrm{m}$. (b) Cumulative total dendrite length per neuron in somatosensory cortex of WT, shRNACuxl, and $C u x 2^{-/}$mice. (c) Dendrite apical and basal length

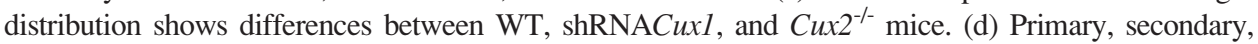
and tertiary dendrite branch numbers in the apical compartment. (e) Primary, secondary, and tertiary dendrite branch numbers in the basal compartment. (f) Length of primary, secondary, and tertiary den-

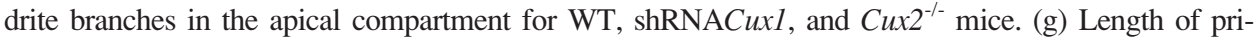
mary, secondary, and tertiary dendrite branches in the basal compartment for WT, shRNACuxl, and Cux $2^{---}$mice. Results represent mean \pm SEM. Control, $n=24 ; C u x 2^{-/-}, n=21$. One-way ANOVA followed by Tukey's post hoc test $* p<0.05$, ** $p<0.01$, *** $p<0.001$ compared to controls. 
a

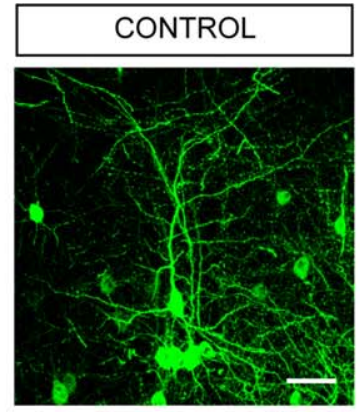

b

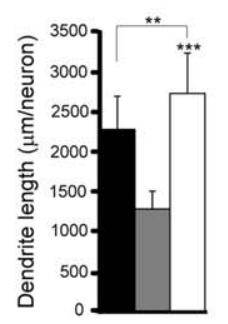

d

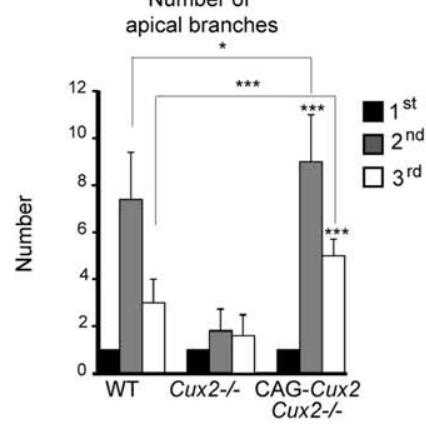

f

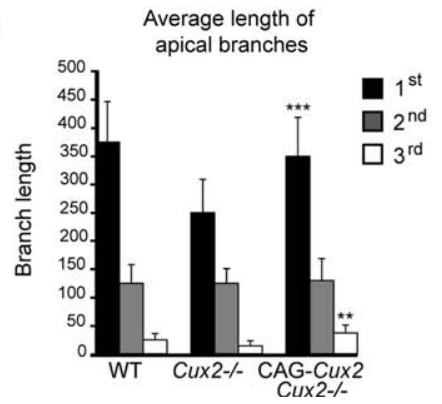

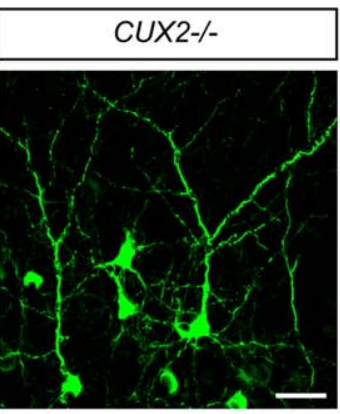

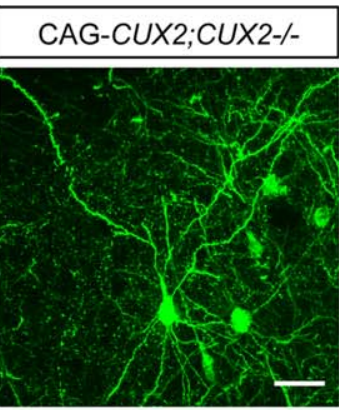

C

Dendrite length
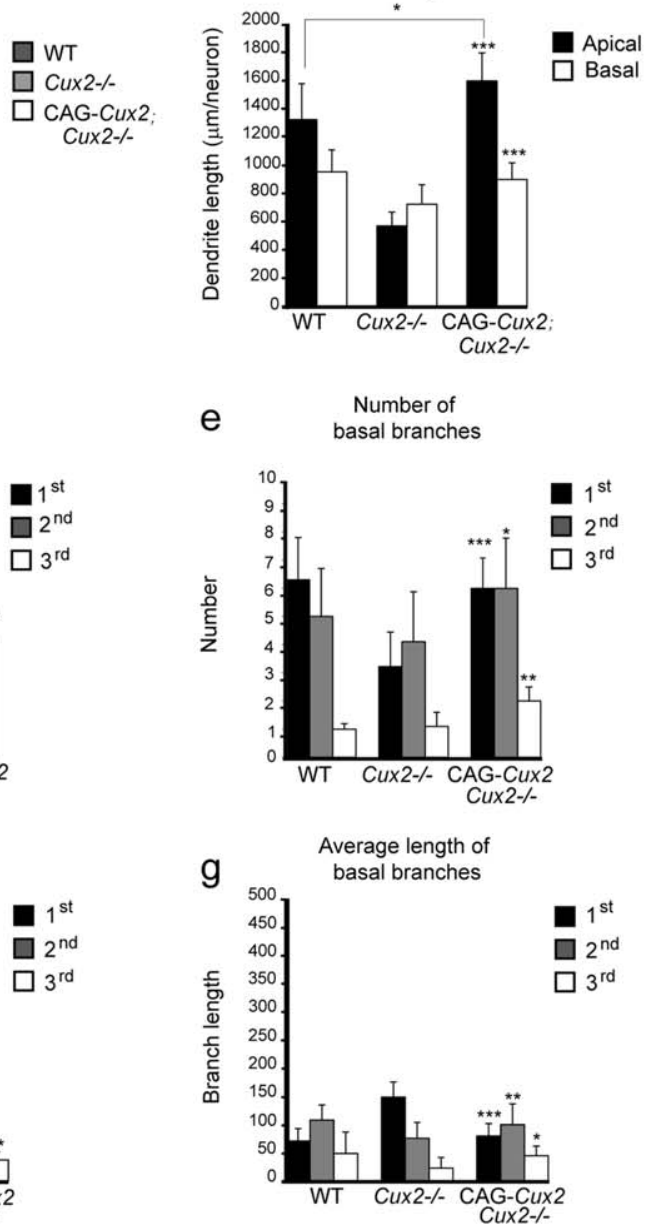

Figure 2 Cux2 overexpression rescue apical dendrite length and branching of $C u x 2^{-/-}$upper layer neurons. $\mathrm{Cu} x 2$ overexpression in $\mathrm{Cux2^{-/ }}$ animals increased apical dendrite complexity compared to $\mathrm{Cux} 2^{-/-}$neurons electroporated with control vector. (a) Representative confocal micrographs showing GFP-expressing neurons of $\mathrm{P} 21$ somatosensory cortex. $C A G-C u x 2$ or $C A G$ empty vectors were coelectroporated with the $C A G-G F P$ construct in control WT or $C u \times 2^{-/-}$animals. Bar $=50 \mu \mathrm{m}$. (b) Cumulative total dendrite length per neuron. (c) Total length of apical and basal branches. (d) Number of apical primary, secondary, and tertiary dendrites. (e) Number of basal primary, secondary, and tertiary dendrites. (f) Average length of primary, secondary, and tertiary apical dendrite branches (g) Average length of primary, secondary, and tertiary basal dendrite branches. Results represent mean \pm SEM. Control $C A G-G F P, n=24 ; C u \times 2^{-/-}, n=21 ; C A G-C u \times 2, n=17$. One-way ANOVA followed by Tukey's post hoc test $* p<0.05$, ** $p<0.01$, *** $p<0.001$. CAG-Cux2; $\mathrm{Cux} 2^{-/-}$is compared to $\mathrm{Cu} \times 2^{-/-}$and WT-control. [Color figure can be viewed in the online issue, which is available at wileyonlinelibrary.com.] 
a

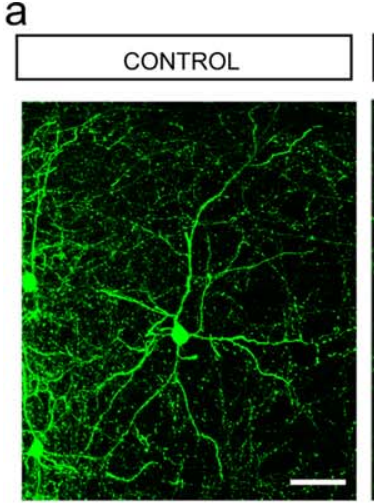

b

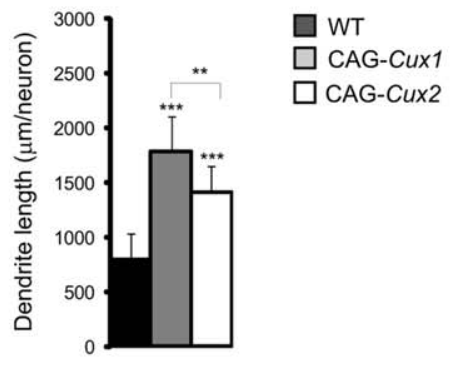

d

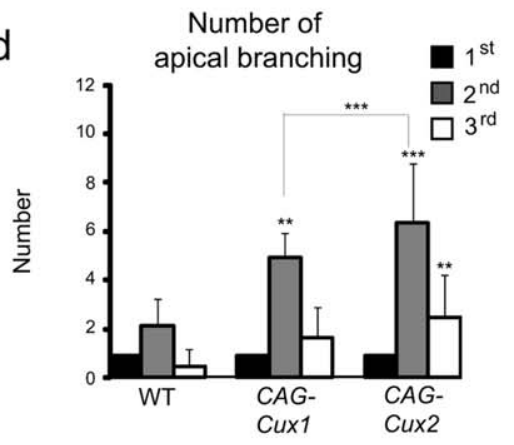

f

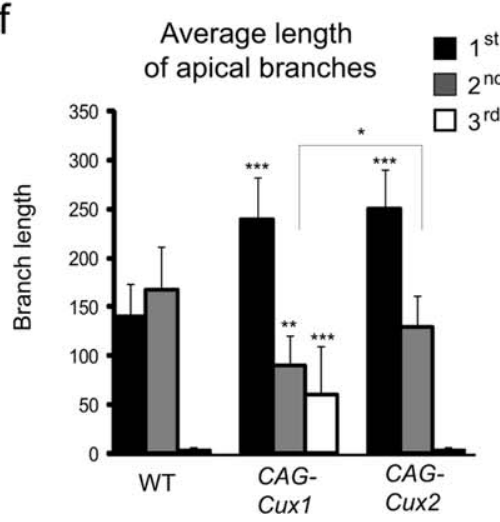

CAG-CUX1

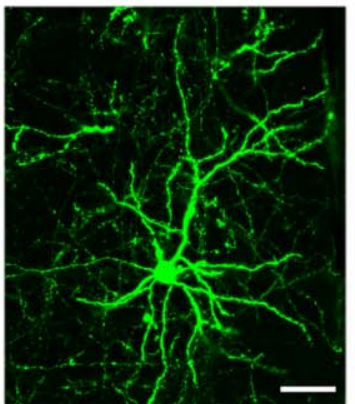

C

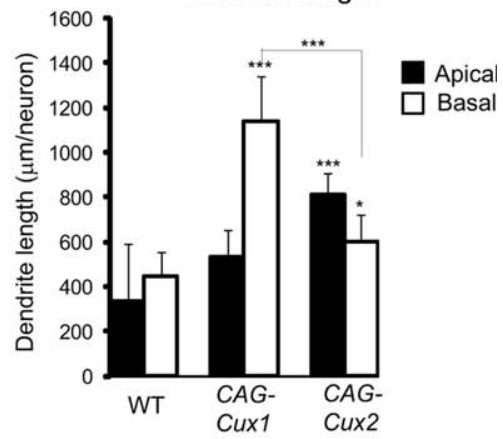

Number of

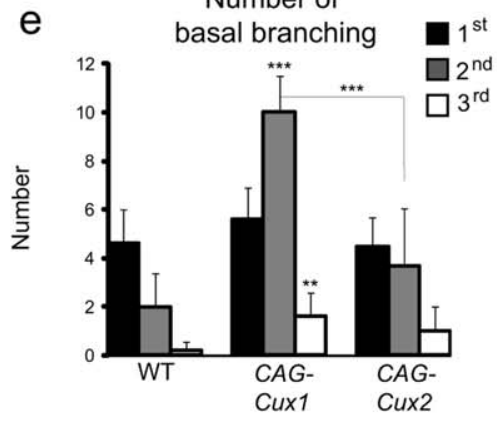

g

Average length of basal branches

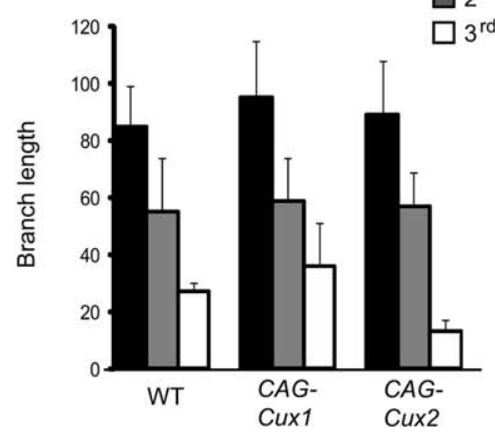

Figure 3. 
neurons [Fig. 1(a,b)], as previously reported (Cubelos et al., 2010). Both apical and basal compartments were affected in shRNACuxl targeted neurons compared to control. However, loss of $C u x l$ function has a stronger effect on basal dendrites compared to loss of Cux2 [Fig. 1(c)]. This was due to a profound decrease in the number of secondary and tertiary apical branches [Fig. 1(d)], whose length did not change [Fig. 1(f)].

\section{Forced Cux1 and Cux2 Expression in Cingulate Neurons Promotes Selective Development of Basal and Apical Dendrites}

To further assess the functions of $C u x$ genes in the apical and basal compartments, we over-expressed Cuxl and $C u \times 2$ in the cingulated cortex of WT animals. Late-born neurons of the cingulate cortex have relatively simple dendritic morphologies (Cubelos et al., 2010) and are, therefore, an appropriate population in which to analyze possible selective effects of Cux proteins in stimulating dendritogenesis. These neurons express both Cuxl and Cux2, with lower Cuxl levels (Nieto et al., 2004; Ferrere et al., 2006). CAG-Cuxl and $C A G-C u x 2$ constructs were electroporated in utero in the dorso-medial ventricle of E15.5 WT embryos, and mice were analyzed at P21. Cuxl or Cux2 overexpression did not affect the correct generation and migration of electroporated cells, as previously observed (Cubelos et al., 2010). Neurons electroporated with $C A G-G F P$ alone served as controls; they had the characteristic morphology of superficial cingulate neurons, with a long apical dendrite that often branched into two before reaching the molecular layer (layer I), and with relatively few apical secondary branches [Fig. 3(a,d)]. The basal branches developed a more complex arbor, with a larger number of primary and secondary projections [Fig. 3(a)]. Nonetheless basal branches in the cingulate, presented less complexity than those of the somatosensory cortex [compare Figs. 1(c) and 3(c)]. Quantitative analysis of total dendritic length showed that both Cuxl and Cux2 overexpression induced a significant increase in neuron complexity [Fig. 3(a,b)]. Detailed examination of branching number and average length of specific dendrites showed that Cuxl overexpression increased the number of secondary and tertiary branches in apical and basal compartments [Fig. 3(d,e)], indicating a general effect on stimulation of dendritogenesis. Nonetheless, the increase in basal dendrites was more marked [Fig. 3(e)]. Neurons with high Cux2 levels behaved in a different manner, with a greater increase in the number of apical than of basal branches. Elevated expression of Cuxl and Cux2 affected the average length of apical branches but not of basal [Fig. 3(f,g)]. The results, thus, confirmed the stronger effects of Cux2 in the apical branches observed in the $C u x 2^{-/-}$mice, and showed the distinct effects compared to $C u x l$, which also strongly stimulates basal dendrites. The results strongly suggest differential effects of Cuxl and Cux2, which contributes to understand their complementary functions. They indicate that in WT cortex, the overlapping $C u x$ expression patterns contributes to the generation of functionally diversified populations of layer II-III neurons.

\section{DISCUSSION}

We show that Cuxl and Cux2 have differential effects on dendrite development of cortical layer IIIII neurons. While Cuxl modulates more markedly the basal dendritic compartment, Cux2 shows a more selective effect on apical dendrites. The distinct effects reported herein contribute to clarify the relevance of the coexpression and functions of Cuxl and

Figure 3 Cuxl and Cux 2 overexpression regulate dendrite length and branching in cingulate cortex. (a) Representative confocal micrographs showing GFP-expressing neurons. Neuron morphology was analyzed in the P21 cingulate cortex after in utero electroporation of E15.5 cortical precursors. CAG-Cuxl, CAGCux2, or $C A G$ empty vectors were coelectroporated with the $C A G-G F P$ construct. $C u x 1$ or $C u x 2$ overexpression increased dendrite complexity of cingulate neurons compared with neurons electroporated with control vector. Bar $=50 \mu \mathrm{m}$. (b) Cumulative total dendrite length per neuron. (c) Total length of apical and basal branches differed when $C u x 1$ or $C u x 2$ was overexpressed. (d) Number of apical primary, secondary and tertiary dendrites. (e) Number of basal primary, secondary, and tertiary dendrites. (f) Length of primary, secondary, and tertiary dendrite branches in the apical compartment after CAG-Cuxl or CAG-Cux2 overexpression in neurons. (g) Length of primary, secondary, and tertiary dendrite branches in the basal compartment after $C A G-C u x l$ or $C A G-C u x 2$ overexpression in neurons. Results represent mean $\pm \mathrm{SEM}$. Control $C A G$-GFP, $n=14$; $C A G$-Cuxl, $n=12$; CAG-Cux2, $n=17$. One-way ANOVA followed by Tukey's post hoc test $* p<0.05$, ** $p<0.01, * * * p<0.001$ compared to controls. [Color figure can be viewed in the online issue, which is available at wileyonlinelibrary.com.] 


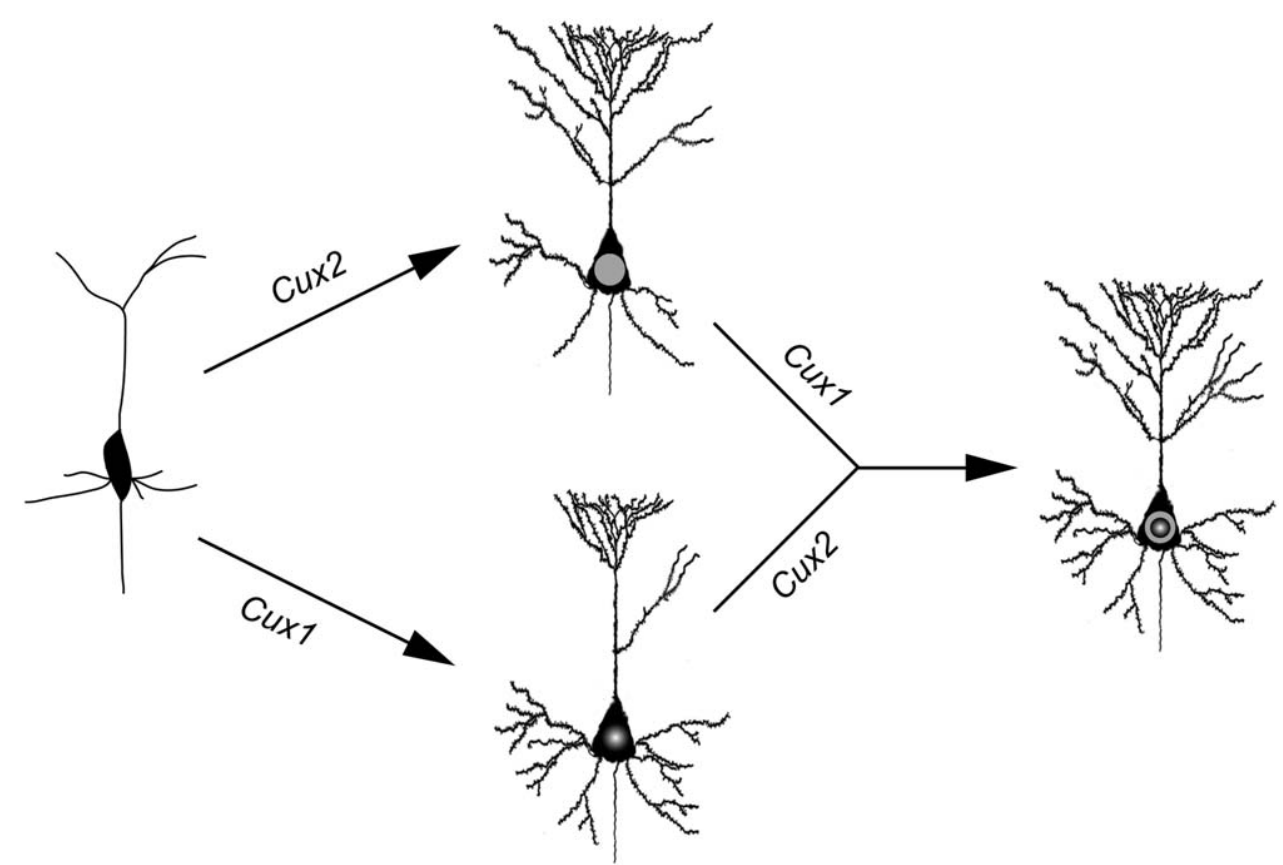

Figure 4 Cux proteins selectively target apical and basal dendritic domains of layer II-III cortical neurons. Scheme summarizing the additive and complementary functions of Cuxl and Cux2. During brain development, $C u x l$ expression has a stronger effect on the development of basal processes while $C u \times 2$ preferentially contributes to apical dendrite differentiation. Coexpression of the two genes determines the final dendritic arbor of layer II-III neurons.

Cux2 in layer II-III neurons [Fig. 4], where they determine the final complexity of the dendritic arbor of these neurons (Cubelos et al., 2010).

Recent studies identified the importance of apical and basal dendrite regulation in the physiology and development of neuronal circuits; many of these reports established a link between these mechanisms and pathways implicated in human autism disorders. Several features of neuron function are related directly to dendrite polarity. Dendrite topography selects the number and type of presynaptic axons, which have a layered organization in the cortex, for the simple reason that it restricts accessibility to potential synapses. Axon contacts can even discriminate between basal and apical domains of the same neuron, probably by recognizing polarized signals (Mainen and Sejnowski, 1996; Petreanu et al., 2009; Jia et al., 2010). The modulation of apical and basal morphologies also influences input computation of the neuron and modulates neuron excitability and plasticity as propagation of electrical signals also differs in distal apical and basal domains (Jia et al., 2010; Branco and Hausser, 2011). The combination and modulation of $C u x 1$ and $C u x 2$ expression allows layer II-III neuron differentiation into further specialized subpopulations and might contribute to the formation and function of selected intra- cortical subnetworks (Yoshimura and Callaway, 2005; Yoshimura et al., 2005; Yassin et al., 2010). It might also explain the reported distinct complexity of basal dendrites of layer II-III neurons in the different areas of murine and human cortex (Elston, 2003; Ballesteros-Yanez et al., 2006).

The mechanisms that regulate dendrites downstream of Cux, or downstream of other transcription factors involved in the subclass specific dendrite determination are mostly unknown (Grueber et al., 2005; Parrish et al., 2007; Cline and Haas, 2008; Fame et al., 2011). They are likely linked to one or several cytoskeleton, membrane receptors, and signaling proteins reported to regulate dendritic differentiation (Kuijpers and Hoogenraad, 2011; Kulkarni and Firestein, 2012). Of particular interests are the selective effects of $\mathrm{Cux} 2$ in apical dendrites. Some of above mentioned cellular mechanisms act selectively on apical or basal dendrites. Brain-derived neurotrophic factor and neurotrophin-3 (NT-3) for example, showed to enhance elongation of basal dendrites of layer II-III pyramidal neurons in the rat somatosensory cortex, but did not affected the apical processes (Baker et al., 1998; Niblock et al., 2000). Signaling of Sema3A through its receptor Nrp1/PlexA4 controls basal dendritic arborization 
in layer $\mathrm{V}$ cortical neurons, providing another example of pathways stimulating polarized growth (Fenstermaker et al., 2004; Tran et al., 2009). The kinase TAOK2 mediates this effect through the interaction with Nrp1 molecules bound to Sema3A. TAOK2 is encoded by the autism spectrum disorder (ASD) susceptibility gene TAOK2 (de Anda et al., 2012).

The study by de Anda et al. adds to previous work showing the importance of dendritic development in ASD and to recent work showing a link with developmental failures in apical and basal cell compartments. The Angelman syndrome protein ubiquitin-protein ligase E3A (Ube3a) promotes apical dendrite outgrowth by regulating the asymmetric distribution of the Golgi apparatus (Miao et al., 2013). Epac2 rare coding variants are found in human subjects diagnosed with autism. Epac2 is a guanine nucleotide exchange factor (GEF) for the Ras-like small GTPase Rap necessary to maintain the basal compartment of layer II-III neurons of mice and humans (Srivastava et al., 2012b); and for learning and normal social interaction in mice (Yang et al., 2012; Srivastava et al., 2012a). These and other possible mechanisms mediating the selective targeting of apical domains by $C u x 2$, or both apical and basal domains by $C u x l$, suggest a linkage of $C u x$ genes with ASD. This requires further investigations also encouraged by studies showing that, in man, CUXI mediates abnormal transcriptional activation of two intronic single-nucleotide polymorphisms of ENGRAILED2 linked to ASDs (Choi et al., 2012).

Our data demonstrates differential effects of Cuxl and Cux2 in dendritic development. These distinct functions might serve to specialize layer IIIII neurons of the mammalian cortex as well as other neuronal subpopulations expressing Cuxl or Cux2. The importance of apical and basal compartments for the high functions of the brain predicts that the molecular and cellular programs mediated by $C u x$ genes contribute to the precise organization of cortical networks; it also suggests that mutations affecting $C u x$ pathways might be implicated in autism disorders.

We thank R. Gutiérrez and A. Morales for excellent technical assistance and C. Mark for editorial assistance.CGB, GMEO BC performed the experiments. BC and $\mathrm{MN}$ designed, and analysed data. MN and BC obtained funding. All authors participated in the preparation of the manuscript. All authors approved the final manuscript.

Disclosure: The authors declare that they have no competing interests.

\section{REFERENCES}

Baker RE, Dijkhuizen PA, Van Pelt J, Verhaagen J. 1998. Growth of pyramidal, but not non-pyramidal, dendrites in long-term organotypic explants of neonatal rat neocortex chronically exposed to neurotrophin-3. Eur J Neurosci 10:1037-1044.

Ballesteros-Yanez I, Benavides-Piccione R, Elston GN, Yuste R, DeFelipe J. 2006. Density and morphology of dendritic spines in mouse neocortex. Neuroscience 138: 403-409.

Barnes AP, Solecki D, Polleux F. 2008. New insights into the molecular mechanisms specifying neuronal polarity in vivo. Curr Opin Neurobiol 18:44-52.

Bi X, Lynch G, Zhou J, Gall CM. 2001. Polarized distribution of alpha5 integrin in dendrites of hippocampal and cortical neurons. J Comp Neurol 435:184-193.

Branco T, Hausser M. 2011. Synaptic integration gradients in single cortical pyramidal cell dendrites. Neuron 69: 885-892.

Choi J, Ababon MR, Matteson PG, Millonig JH. 2012. Cutlike homeobox 1 and nuclear factor $\mathrm{I} / \mathrm{B}$ mediate ENGRAILED2 autism spectrum disorder-associated haplotype function. Hum Mol Genet 21:1566-1580.

Cline H, Haas K. 2008. The regulation of dendritic arbor development and plasticity by glutamatergic synaptic input: A review of the synaptotrophic hypothesis. J Physiol 586:1509-1517.

Cubelos B, Nieto M. 2010. Intrinsic programs regulating dendrites and synapses in the upper layer neurons of the cortex. Commun Integr Biol 3:483-486.

Cubelos B, Sebastian-Serrano A, Beccari L, Calcagnotto ME, Cisneros E, Kim S, Dopazo A, et al. 2010. Cux1 and Cux2 regulate dendritic branching, spine morphology, and synapses of the upper layer neurons of the cortex. Neuron 66:523-535.

Cubelos B, Sebastian-Serrano A, Kim S, Moreno-Ortiz C, Redondo JM, Walsh CA, Nieto M. 2008a. Cux-2 controls the proliferation of neuronal intermediate precursors of the cortical subventricular zone. Cereb Cortex 18:17581770 .

Cubelos B, Sebastian-Serrano A, Kim S, Redondo JM, Walsh C, Nieto M. 2008b. Cux-1 and Cux-2 control the development of Reelin expressing cortical interneurons. Dev Neurobiol 68:917-925.

de Anda FC, Rosario AL, Durak O, Tran T, Graff J, Meletis K, Rei D, et al. 2012. Autism spectrum disorder susceptibility gene TAOK2 affects basal dendrite formation in the neocortex. Nat Neurosci 15:1022-1031.

Elston GN. 2003. Cortex, cognition and the cell: New nsights into the pyramidal neuron and prefrontal function. Cereb Cortex 13:1124-1138.

Fame RM, MacDonald JL, Macklis JD. 2011. Development, specification, and diversity of callosal projection neurons. Trends Neurosci 34:41-50.

Feldman DE. 2012. The spike-timing dependence of plasticity. Neuron 75:556-571. 
Fenstermaker V, Chen Y, Ghosh A, Yuste R. 2004. Regulation of dendritic length and branching by semaphorin $3 \mathrm{~A}$. J Neurobiol 58:403-412.

Ferrere A, Vitalis T, Gingras H, Gaspar P, Cases O. 2006. Expression of Cux-1 and Cux-2 in the developing somatosensory cortex of normal and barrel-defective mice. Anat Rec A Discov Mol Cell Evol Biol 288:158-165.

Grueber WB, Yang CH, Ye B, Jan YN. 2005. The development of neuronal morphology in insects. Curr Biol 15: R730-R738.

Jan YN, Jan LY. 2010. Branching out: Mechanisms of dendritic arborization. Nat Rev Neurosci 11:316-328.

Jia H, Rochefort NL, Chen X, Konnerth A. 2010. Dendritic organization of sensory input to cortical neurons in vivo. Nature 464:1307-1312.

Kuijpers M, Hoogenraad CC. 2011. Centrosomes, microtubules and neuronal development. Mol Cell Neurosci 48: 349-358.

Kulkarni VA, Firestein BL. 2012. The dendritic tree and brain disorders. Mol Cell Neurosci 50:10-20.

Luong MX, van der Meijden CM, Xing D, Hesselton R, Monuki ES, Jones SN, Lian JB, et al. 2002. Genetic ablation of the $\mathrm{CDP} / \mathrm{Cux}$ protein $\mathrm{C}$ terminus results in hair cycle defects and reduced male fertility. Mol Cell Biol 22:1424-1437.

Mainen ZF, Sejnowski TJ. 1996. Influence of dendritic structure on firing pattern in model neocortical neurons. Nature 382:363-366.

Miao S, Chen R, Ye J, Tan GH, Li S, Zhang J, Jiang YH, et al. 2013. The Angelman syndrome protein Ube3a is required for polarized dendrite morphogenesis in pyramidal neurons. J Neurosci 33:327-333.

Niblock MM, Brunso-Bechtold JK, Riddle DR. 2000. Insulin-like growth factor I stimulates dendritic growth in primary somatosensory cortex. J Neurosci 20:4165-4176.

Nieto M, Monuki ES, Tang H, Imitola J, Haubst N, Khoury SJ, Cunningham J, Gotz M, Walsh CA. 2004. Expression of Cux-1 and Cux-2 in the subventricular zone and upper layers II-IV of the cerebral cortex. J Comp Neurol 479: 168-180.

Parrish JZ, Emoto K, Kim MD, Jan YN. 2007. Mechanisms that regulate establishment, maintenance, and remodeling of dendritic fields. Annu Rev Neurosci 30:399-423.

Petreanu L, Mao T, Sternson SM, Svoboda K. 2009. The subcellular organization of neocortical excitatory connections. Nature 457:1142-1145.
Polleux F, Morrow T, Ghosh A. 2000. Semaphorin 3A is a chemoattractant for cortical apical dendrites. Nature 404: 567-573.

Shen K, Scheiffele P. 2010. Genetics and cell biology of building specific synaptic connectivity. Annu Rev Neurosci 33:473-507.

Srivastava DP, Jones KA, Woolfrey KM, Burgdorf J, Russell TA, Kalmbach A, Lee H, et al. 2012a. Social, Communication, and Cortical Structural Impairments in Epac2-Deficient Mice. J Neurosci 32:11864-11878.

Srivastava DP, Woolfrey KM, Jones KA, Anderson CT, Smith KR, Russell TA, Lee H, et al. 2012b. An autismassociated variant of Epac2 reveals a role for Ras/Epac2 signaling in controlling basal dendrite maintenance in mice. PLoS Biol 10:e1001350.

Tabata H, Nakajima K. 2001. Efficient in utero gene transfer system to the developing mouse brain using electroporation: Visualization of neuronal migration in the developing cortex. Neuroscience 103:865-872.

Tada T, Sheng M. 2006. Molecular mechanisms of dendritic spine morphogenesis. Curr Opin Neurobiol 16:95101.

Tran TS, Rubio ME, Clem RL, Johnson D, Case L, TessierLavigne M, Huganir RL, et al. 2009. Secreted semaphorins control spine distribution and morphogenesis in the postnatal CNS. Nature 462:1065-1069.

Yang Y, Shu X, Liu D, Shang Y, Wu Y, Pei L, Xu X, et al. 2012. EPAC null mutation impairs learning and social interactions via aberrant regulation of miR-124 and Zif268 translation. Neuron 73:774-788.

Yassin L, Benedetti BL, Jouhanneau JS, Wen JA, Poulet JF, Barth AL. 2010. An embedded subnetwork of highly active neurons in the neocortex. Neuron 68: 1043-1050.

Yoshimura Y, Callaway EM. 2005. Fine-scale specificity of cortical networks depends on inhibitory cell type and connectivity. Nat Neurosci 8:1552-1559.

Yoshimura Y, Dantzker JL, Callaway EM. 2005. Excitatory cortical neurons form fine-scale functional networks. Nature 433:868-873.

Zimmer C, Tiveron MC, Bodmer R, Cremer H. 2004. Dynamics of $\mathrm{Cux} 2$ expression suggests that an early pool of SVZ precursors is fated to become upper cortical layer neurons. Cereb Cortex 14:1408-1420. 